\title{
A Mobile Cloud-supported e-Rehabilitation Platform for Brain-Injured Patients
}

\author{
Angel Ruiz-Zafra, Manuel Noguera, \\ Kawtar Benghazi, Jose Luis Garrido \\ Dpto. Lenguajes y Sistemas Informáticos \\ University of Granada \\ Granada, Spain \\ anruza@correo.ugr.es,mnoguera@ugr.es, \\ benghazi@ugr.es,jgarrido@ugr.es
}

\author{
Gustavo Cuberos Urbano \\ Dpto. Personalidad, Evaluación y \\ Tratamiento Psicológico \\ University of Granada \\ Granada, Spain \\ gcuberos@ugr.es
}

\author{
Alfonso Caracuel \\ Dpto. Psicología Evolutiva y de la \\ Educación \\ University of Granada \\ Granada, Spain \\ acaracuel@ugr.es
}

\begin{abstract}
Currently, brain injury rehabilitation programs aid patients to regain their daily living skills. In these programs, each patient has to repeatedly perform a wide range of rehabilitation exercises under the in situ supervision of health professionals, normally, in a medical center or (unusually) at home, during training sessions. This model entails frequent displacements, either of professionals or patients for the sessions to be supervised. A Home-based and mobile e-rehabilitation platform by which patients could be effectively supervised, remotely and asynchronously, during the performance of the prescribed exercises can be an alternative to this model that would reduce number of displacements. Mobile devices and novel computation paradigms such as Cloud Computing can be used to support cooperation and interaction between health professionals, patients and their relatives in order to come up with home-based e-rehabilitation programs for brain-injured patients where medical records about patient performances are easily accessible and stored. This paper describes CloudRehab, a customizable home-based e-rehabilitation platform for brain-injured patients that make use of mobile devices, commercial heart rate sensors, Web and Cloud Computing technologies, which has been validated with several patients.
\end{abstract}

Keywords- brain-injured; e-rehabilitation; mobile devices; cloud computing; cloud rehab;

\section{INTRODUCTION}

Acquired Brain Injuries (ABI) are normally caused by accidents, cerebrovascular diseases, tumors, anoxia, etc. Only focusing in Traumatic Brain Injuries (TBI), a common cause of $\mathrm{ABI}[1]$, more than 5.5 million people are living with any kind of disability for TBI in USA and Europe [2]. Brain-injured patients need a full time monitoring and long rehabilitation time, which makes TBI health-care rather costly. According to [3], the rehabilitation cost for each brain-injured patient can reach near $14.000 €$ in the first two years of disease and $36.000 €$ in the next two years. It also depends on the country, the average cost in USA is 85,000\$[4], and whether the rehabilitation is covered by public or private insurance companies. A high percentage of this medical cost derives from the custom medical assistance (nurses, rehabilitation sessions, therapists, etc.) required.
The goal of brain injuries rehabilitation programs is to aid the patient to achieve the maximum degree of return to their previous normal lifestyle. Brain injury rehabilitation is normally carried out in a medical center. It has the advantage for the patient that he/she has eventual access to suitable medical supplies during the rehabilitation sessions, as well as close supervision and guidance by a health professionals who ensure patients perform correctly the rehabilitation exercises.

The rehabilitation sessions are performed several times a week. This implies that the patient has to move to the medical center every week over a long period of time (from months to years), which increases the rehabilitation costs.

Home-based rehabilitation by remote supervision of exercises would reduce the need for face-to-face rehabilitation sessions with health professionals and patients. Technology may reduce displacements of patients to the hospital, some premature discharges of patients and health-care costs. Likewise, some devices allow a proper monitoring of patient's performance of activities of daily living (ADL). Although home-based rehabilitation could reduce final rehabilitation costs, there is a requirement for effectiveness; it must be assured the correct performance of the rehabilitation exercises without a direct supervision, just using medical feedback. Another important requirement is the existence of appropriate tool support to manage rehabilitation sessions and friendly access (delocalized, everywhere, everytime) to patient information records.

In this paper we present a brain-injury home-based erehabilitation platform that makes use of mobile devices and that helps patients perform rehabilitation exercises in a proper way. All these exercises are previously designed by the therapist, who can also provide feedback about patient's performance. It provides a web-accessible application based on cloud computing technology. The remainder of this paper is organized as follows. Section II shows the related work, some e-rehabilitation platforms/system for brain-injured patients. The platform is described in the Section III. Section IV provides the results of a validation study about the platform in a single research case. Conclusions and future work are presented in Section V. 


\section{RELATED WORK}

The growth of mobile computing has motivated an increment in the number of home-based rehabilitation systems. As there are many diseases that need rehabilitation, different specialized rehabilitation systems have appeared recently.

Several general purpose systems based on human motion tracking for rehabilitation exercises have been proposed [4][5]. These kinds of systems are usually based on wireless body sensors such as accelerometers [6][7] that provide some feedback (normally stored in the body sensor) about patient movements. When the patients perform a rehabilitation exercise a technician has to save this information from the body sensor to personal computer or laptop. This raw information is to be checked by health professional at a later moment.

Alternatively, there exist rehabilitation systems for specific diseases and purposes, such as [8], for upper limb and knee diseases [11], and rehabilitation systems based on mobile technologies for patients with cardiac diseases [9][10].

As for e-rehabilitation systems for brain-injured patients, there are different platforms developed by research groups (public) and companies (private). It is worth pointing out EuroPaNet [12], a European public telecare project for TBI erehabilitation that is being used currently in different countries (Italy, Belgium, Spain). It makes use of client-server applications where patients perform rehabilitation exercises using interactive software. The platform provides medical assistance such as email exchange or real-time videoconferencing between the patients and health professionals, but does not provide any kind of health feedback (heart rate, blood pressure). PREVIRNEC [13] is a public project for e-rehabilitation patients with $\mathrm{ABI}$ where the patients are to carry out different activities, such as games or virtual exercises, through a web browser designed by the therapist. The result of these tasks is stored in a web server and can be accessed by doctors. As EuropaNet, PREVIRNEC provides a real-time videoconferencing facility, but does not provide health feedback.

TEREHA [14] platform has been designed and developed by different private companies, such as Everis and NeuroRHB. It consists of three modules for motor, cognitive and psychological rehabilitation and makes use of social networks to aid brain-injured patients. Patients use friendly applications and their relatives can follow patients' evolution in the social networks.

\section{Cloud RehaB: Platform OVERVIEW}

\section{A. Context Scenario}

Brain-injured patients have to perform periodically rehabilitation exercises designed by professionals to regain their motor, language, cognitive and/or daily living skills. This approach usually entails that patients have to move to the hospital to perform the exercise under medical supervision, which represents an important drawback for this kind of patients.

The main goal of CloudRehab is to provide a remote supervision platform for health professionals (doctors,

\footnotetext{
Identify applicable sponsor/s here. (sponsors)
}

occupational therapists, physiologists) who can use the platform to train daily activities, increase independence and reduce patient displacements. To achieve this, professionals design training sessions and help patients learn how to perform them, which is video-recorded.

In each training session different information is stored: the performance of the exercise (video recorded by means of the frontal camera of the mobile device) together with the heart rate values obtained from a chest strap Bluetooth sensor [15]. This information is simultaneously stored in the mobile device and in a remote cloud [16]. This way, the video sessions and the heart rate values recorded can be accessed anywhere and anytime. This is an important feature, since it enables asynchronous interaction and work with the platform of patients and health professionals, which in turn, permits that certain activities, such as eating, can be performed fitting the usual schedule of the patient.

Only a mobile device with Bluetooth and a frontal camera, as well as Internet connection (for real-time monitoring and cloud storage of session information) is needed. Figure 1 provides an overview of the platform. Figure 1 shows an overview of CloudRehab.

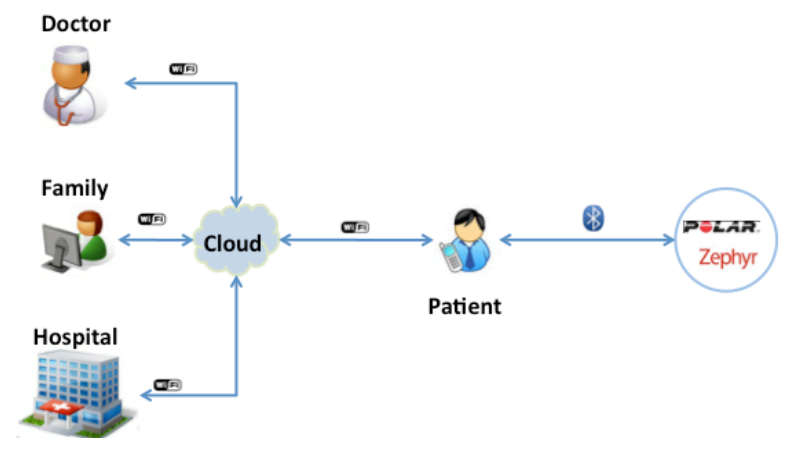

Figure 1. CloudRehab Platform Overview

\section{B. Platform Applications}

The platform is made up of, among others, two main applications:

\section{1) CloudRehab Web Site}

It consists of a web application that can be used by any user of the system (health professionals, patient's relatives and patients). Although all of them use the same web application, each type of user has its own interface and restricted access to different sets of functionalities. The different functionalities provided are:

- Manage patient information: A health professional can register into the system his/her owns patients and define the medical and personal information.

- Manage session's patient: One session is made up of a training video of a patient performing an activity in a training process, a set of audio and image files used when the heart rate value of the patient reach a certain value, the recorded video of the session, the heart rate values, and alerts to be triggered, among others. The professional can define new sessions with new training activities and, once the patient has completed them, 
review session information to evaluate patient progress.

- $\quad$ Review information generated by the patient such as completed sessions (recording video, recording audio, heart rate records) (Figure 2), heart rate between two different dates or charts (sectors, bars) of the patient's progress over time (Figure 3).

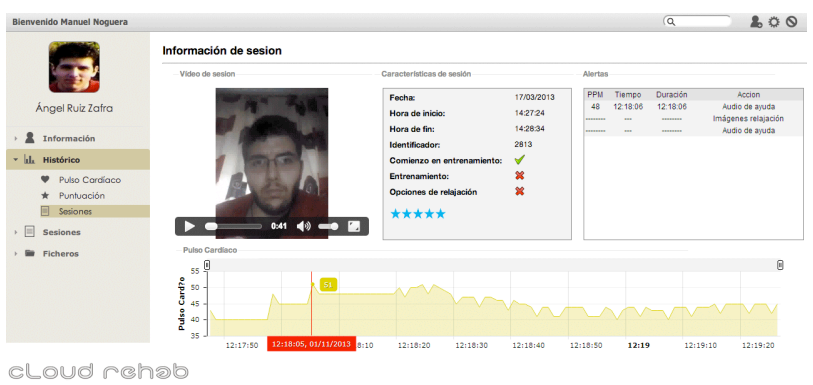

Figure 2. Information of Completed Session

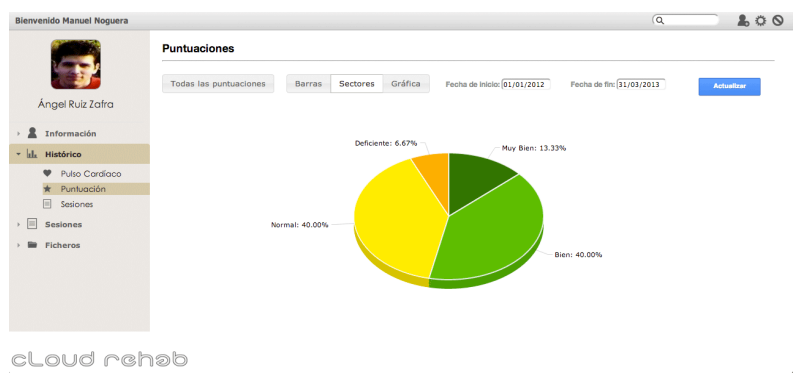

Figure 3. Sector chart of patient's medical evolution

- Real-time Session Monitoring: Professionals and patients' families can view the heart rate records and the recording video in real time.

\section{2) CloudRehab Mobile Application}

A mobile application (app) based on Android OS that allows the training session of an exercise to be played and recorded while obtaining heart rate using a chest strap sensor [15] to monitor patient's stress level.

The application divides the device screen into two different parts. The upper part shows the images captured by the frontal camera, while the bottom part of the screen displays the training video previously recorded with the supervision of a therapist (Figure 4 - Left). This provides to the patient a useful real-time feedback about how to perform the exercise correctly and how he/she is approaching to the intended model.

If the application detects that the heart rate signal of the patient has reached the threshold define by the professionals, the application plays an information sound and shows a pop-up dialog with different options (i.e., review the video session by steps, play custom help audio or view relaxing images) in order to aid the patient to reduce his/her stress level.

The mobile application also provides other functionalities such as real-time heart rate sensor detection, change screen mode (only video, camera and video or only camera mode) and statistical information display (Figure 4 right), among others.

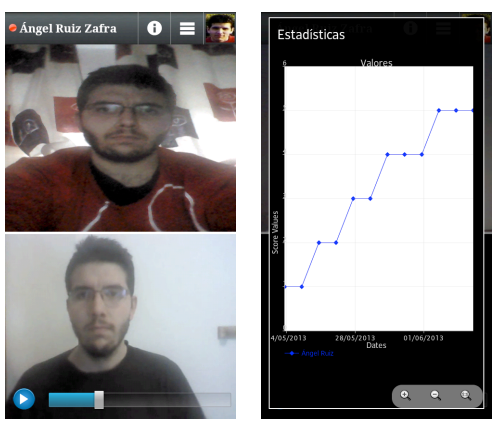

Figure 4. Video recoding/playing(left) and statistical score (right)

\section{EFFECTIVENESS OF CLOUD REHAB PLATFORM USING A SINGLE CASE RESEARCH DESIGN}

The Cloud Rehab platform was applied in a pilot study with three ABI patients and with satisfactory results in all cases concerning the rehabilitation of skills and patient satisfaction. The main and most complete study was carried out with a 51 years old male who suffered an ischemic stroke 2 years ago. The participant had a chronic hemiplegia of right limbs, a common long-term consequence of stroke [17]. He was undergoing a therapy for gait rehabilitation in a public hospital. A daily living activity that was not an aim of his therapy was chosen for the current study. The target behaviour was "cutting and eating steaks or chunks of meat or fish using the right arm". This behaviour was not present in the repertoire of the participant and was conducted with the help of a relative (for cutting food pieces) and the unaffected limb (prick with fork and put in his mouth the pieces).

To study the effectiveness of the Cloud Rehab platform a single case study design with two phases (baseline and treatment) was conducted. During the baseline phase ( 8 days) the participant was asked to record himself while doing the target behaviour the best he could do. Subsequently, in the treatment phase ( 8 days), the participant performed and recorded the behaviour using the platform.

To assess the patient's performance, two therapists visualized and rated each video from the platform separately. Previously, a professional board defined those components of the target behavior (see criteria in Table 1) that might be rated during assessment. Performance was rated using an ordinal scale from 1 to 3 (1=poor, $2=$ medium, $3=$ good $)$.

For e-rehabilitation tools and single case research design [18] inter-rater reliability is a key feature. An analysis using the Kappa statistic was performed in order to determine consistency among raters for the seven criteria applied to patient's performance. The Spearman Rho coefficient was used for determining total score agreement. Both statistics range from 0 to 1.0 where large numbers mean better reliability, while values near zero suggests that agreement is attributable to chance alone. The inter-rater reliability for the raters resulted in Spearman Rho $=0.94(\mathrm{p}<0.001)$ and Kappa indexes ranging from 0.522 and 0.901 (see table 1). These findings show high concordance between observers and well functioning of the measurement system. However, criterion \#4 ('Arm does not cross the midline of the trunk') might be re-defined in order to achieve better agreement between raters. 
TABLE I. INTER-RATER RELIABILITY ANALYSIS OF MEASUREMENT CRITERIA

\begin{tabular}{|c|l|c|c|}
\hline$\#$ & \multicolumn{1}{|c|}{ Criteria to measure participant' performance } & Kappa & $\boldsymbol{p}^{*}$ \\
\hline 1 & Keep the trunk erect & 0.790 & 0.000 \\
\hline 2 & Keep shoulders symmetrical & 0.661 & 0.001 \\
\hline 3 & $\begin{array}{l}\text { Not replace elbow movement by shoulder } \\
\text { movements }\end{array}$ & 0.813 & 0.000 \\
\hline 4 & Arm does not cross the midline of the trunk & 0.522 & 0.003 \\
\hline 5 & Properly hold the fork & 0.696 & 0.000 \\
\hline 6 & $\begin{array}{l}\text { Bring the fork to his mouth to eat and not the } \\
\text { other }\end{array}$ & 0.901 & 0.000 \\
\hline 7 & $\begin{array}{l}\text { Releasing and then take the fork down unaided } \\
\text { by the unaffected upper limb }\end{array}$ & 1 & 0.000 \\
\hline
\end{tabular}

Effectiveness of CloudRehab platform was based on statistical analysis of change between baseline and intervention phases. The two standard deviation band method was applied [19]. First, mean $(\bar{X}=8.72)$ and standard deviation $(\mathrm{SD}=0.795)$ of the baseline were calculated. Second, two dotted lines were plotted at two points (mean minus $2 S D$ and mean plus $2 S D$ ) (see Figure 5). When at least two consecutive data points in the treatment phase fall outside of the two-standard deviation range, then a significant change in performance has occurred across the two phases [20].

Participant was also asked to mark his satisfaction with the intervention every day of the treatment phase. Average satisfaction level was 3.75 out of 5 (ranging from 1 to 5).

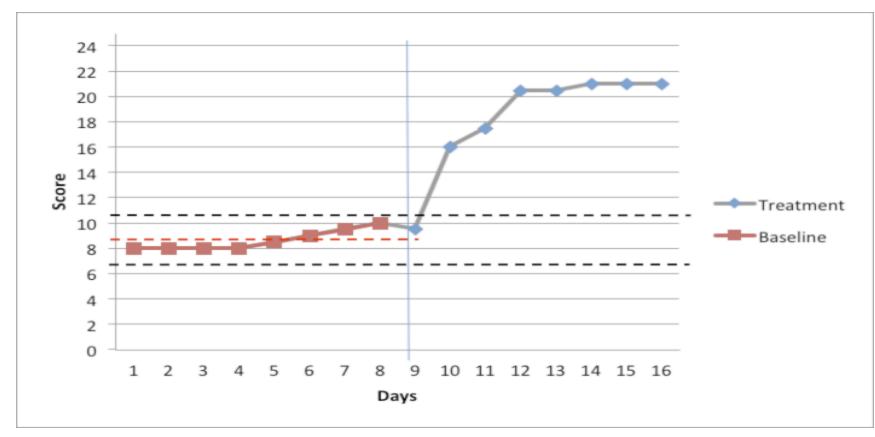

Figure 5. Total score of everyday participant' performance in baseline and treatment phases. Black dotted lines show the two standard deviation ban

\section{CONCLUSIONS AND FUTURE WORK}

This paper describes CloudRehab, a web e-rehabilitation platform for brain-injured patients using mobile devices, commercial heart rate sensors and cloud technology. The platform has been tested with brain-injured patients with satisfactory results in terms of rehabilitation effectiveness while reducing the number of displacements and ease of use. From the point of view of health professionals, they can now count on a useful tool to monitor patients' rehabilitation processes remotely that allow them to work asynchronously.

As future work we are currently working in the design and development of new functionalities as well as the integration of CloudRehab with the information systems and processes of the public health service of our country.

\section{ACKNOWLEDGEMENTS}

This research work has been funded by the CEI BioTIC Granada (20F2/36), Innovation Office from the Andalusian Government (TIN-6600) and the Spanish Ministry of Economy and Competitiveness (TIN2012-38600).

\section{REFERENCES}

[1] The Lancet Neurology. (2010). Traumatic brain injury: time to end the silence. Lancet Neurology, 9, 331. doi:10.1016/S1474-4422(10)70069-7.

[2] http://www.internationalbrain.org/brain-injury-facts/

[3] Braininjury Rehabilitation - a health technology assessment. "National Board of Health, Danish Centre of Health Technology”. pp. 13-14

[4] H. Zhou and H. Hu, 'Human motion tracking for rehabilitation Asurvey”. Biomedical Signal Processing and Control 2007,vol. 3, pp. 1-18

[5] Huiyu Zhou, Housheng $\mathrm{Hu}$ and N. Harris,"Application of wereable inertial sensors in stroke rehabilitation," in Engineering in Medicine and Biology Society EMBS, $27^{\text {th }}$ Annual International Conference of the, 2005, pp. 6825-6828

[6] A.U. Alahakone and S.M.N.A. Senanayake. "A combination of inertial sensors and vibrotactile feedback for balance improvements in therapeutic applications," in Innovate Technologies in Intelligent Systems and Industrial Applications, CITISIA 2009, pp. 5-10

[7] Xiaoping Yun and E.R.Bachmann,"Design,implementation,and experimental Results of a Quaternion-Based Kalman Filter for Human Body Motion Tracking," Robotics, IEEE Transactions on 2006, vol.22, pp.1216-1227

[8] H. Zhou, H.Hu, T. Stone and N. Harris, "Use of multiple wearable inertial sensors in upper limb motion tracking," Med. Eng. Phys. 2007, vol.30, pp. 123-133

[9] Valérie Gay, Peter Leijdekkers, Edward Barin, “A Mobile Rehabilitation Application for the Remote Monitoring of Cardiac Patients after a Heart Attack or a Coronary Bypass Surgery", Proceedings of the 2nd International Conference on PErvasive Technologies Related to Assistive Environments. pp- 2-4

[10] Salminen, J. , Koskinen,E , Kirkeby,O. , Korhonen,I. ,’A home-based care model for outpatient cardiac rehabilitation based on mobile technologies".Pervasive Computing Technologies for Healthcare, 2009. pp. 1-8

[11] Mobolaji Ayoade, Lee Morton, Lynne Baillie, "Investigating the feasibility of a wireless motion capture system to aid in the rehabilitation of total knee replacement patients". Pervasive Computing Technologies for Healthcare (PervasiveHealth), 2011 5th International Conference on, pp. 404-407

[12] T.Roig Rovira, R. Sanchez-Carrión, "EuroPaNet : TeleRehabilitation for TBI patients". European telecare project

[13] J. Solana, C. Caceres, EJ. Gomez, S. Ferrer-Celma, M. Ferre-Bergada, P. Garcia-Lopez, A. Garcia-Molina, A. Garcia-Rudolph, T. Roig, Jm. Tormos, "PREVIRNEC:A new platform for cognitive tele-rehabilitation"

[14] http://www.everis.com/global/en-US/press-room/news/Paginas/terehaintegrated-tele-rehabilitation.aspx

[15] http://www.polar.com/en/products/accessories/Polar_WearLink_transmit ter with Bluetooth

[16] http://www.gnubila.com/

[17] C. Luke, K. J. Dodd, and K. Brock, "Outcomes of the Bobath concept on upper limb recovery following stroke," Clinical Rehabilitation, vol. 18, no. 8, pp. 888-898, 2004

[18] R. L Tate, S. Mcdonald, M. Perdices, L. Togher, R. Schultz, and S. Savage, "Rating the methodological quality of single-subject designs and n-of-1 trials: Introducing the Single-Case Experimental Design (SCED) Scale," Neuropsychological Rehabilitation: An International Journal, vol. 18, no. 4, p. 385, 2008.

[19] M. Bloom, J. Fischer, and J. G. Orme, Evaluating practice. Allyn \& Bacon, 1982

[20] J. M. Gottman and S. R. Leiblum, How to do psychotherapy and how to evaluate it: A manual for beginners. Holt, Rinehart \& Winston, 1974 